Check for updates

Cite this: RSC Adv., 2017, 7, 19621

Received 6th February 2017 Accepted 24th March 2017

DOI: $10.1039 / c 7 r a 01503 h$

rsc.li/rsc-advances

\section{Local controlled release of simvastatin and PDGF from core/shell microspheres promotes bone regeneration in vivo}

\author{
Mingming Yan, (D) a Jiangdong Ni, ${ }^{\star a}$ Hongwei Shen, ${ }^{* b}$ Deye Song, ${ }^{a}$ Muliang Ding ${ }^{a}$ \\ and Jun Huang ${ }^{a}$
}

Simvastatin is demonstrated to be a potent stimulator for bone formation. However, a high dosage of simvastatin is required to induce bone regeneration in systematic administration, which may result in various side effects. Here we achieved the local administration of simvastatin through fabricating core/shell microspheres. Meanwhile, the addition of platelet derived growth factor (PDGF) encapsulated in core/shell microspheres was explored for a better outcome of bone regeneration. The microspheres composed of poly-L-lactide (PLLA) core and alginate shell were formulated via coaxial electrohydrodynamic atomization (CEHDA). The simvastatin and PDGF were incorporated in following models: (1) simvastatin was loaded in the core layer alone (core-simvastatin/shell, M1); (2) simvastatin was encapsulated in the core layer and PDGF was loaded into the shell layer (core-simvastatin/shell-PDGF, M2). Well-formed microspheres with distinct core-shell structures were successfully formulated. In vitro, simvastatin in the core layer displayed a near-zero-order release pattern within 40 days. In contrast, the release profile of PDGF in the shell layer was characterized with a strong initial burst release and being almost depleted in as early as 9 days. The 3(4,5-dimethylthiazol-2-yl)-2,5-diphenyltetrazolium bromide (MTT) assay showed good biocompatibility with PLLA-alginate microspheres. In in vivo experiments, both the M1 and M2 groups showed greater new bone formation, with significantly increased bone mineral density (BMD), the ratio of mineralized bone volume of the defect tissue volume (BV/TV) and trabecula thickness (Tb.Th) of neogenesis bone, while little newly formed bone was found in sham surgery controls and blank microsphere groups. More importantly, the enhancement of bone regeneration was significantly greater in the M2 group. Thus, we suggested that the core/shell microspheres could be a promising delivery system for simvastatin combined with PDGF to improve bone regeneration.

\section{Introduction}

Treatment of segmental bone defects resulting from injury, tumor resection or infection debridement remains a challenge for orthopedic surgeons. It has been reported that about 500000 bone grafting operations are performed per year in the United States. Although autologous bone grafts are still a standard treatment for bone defects, approximately $10 \%$ of patients suffer from the various complications, such as donor site morbidity, chronic pain and infection. ${ }^{1}$ Therefore, the novel approaches ranging from growth factors (GFs) application to mi-RNA interference and bone tissue engineering have been introduced to promote bone regeneration. ${ }^{2,3}$ Among the various growths factors, the bone morphogenetic protein-2 (BMP-2) is

${ }^{a}$ Department of Orthopaedic Surgery, The Second Xiangya Hospital of Central South University, 139 Middle Renmin Road, Changsha 410011, P. R. China. E-mail: hpnjd@163.com; timonzy@126.com

${ }^{b}$ The Center of Medical Research, The Second Xiangya Hospital of Central South University, 139 Middle Renmin Road, Changsha 410011, P. R. China one of the most potent osteogenic growth factor, which has been used in bone repair in clinic and approved by Food and Drug Administration (FDA) for anterior lumbar interbody fusion in skeletally mature patients in 2002..$^{4,5}$ However, the complicated procedures of production, the complexity of equipments required and the long cycle of production needed make the recombinant BMP-2 costly. Moreover, the high dose of BMP-2 has been reported to result in some side effects. ${ }^{6}$ These drawbacks compromise the recombinant BMP-2 as a therapeutic agent for bone regeneration and drive the researchers to explore a small molecule to enhance bone formation. ${ }^{78}$

Since Mundy demonstrated statins, especially simvastatin promoted new bone formation through stimulating BMP-2 gene expression in 1999, ${ }^{9}$ numerous publications have found simvastatin increase expression of osteogenic markers, such as osteocalcin, osteopontin and runt-related transcription factor 2 (RUNX-2) in bone marrow mesenchymal stem cells in vitro. ${ }^{10,11}$ However, systemic administration of simvastatin may compromise its excellent anabolic effects on bone due to the extensive first-pass degradation of simvastatin in liver, which significantly 
reduced the drug concentration in circulation and resulted in little drug accumulation in bone defect. Moreover, the high dosage of simvastatin required to enhance bone regeneration may be detrimental to liver and kidney. ${ }^{12}$ Therefore, local delivery of simvastatin without the first-pass degradation in liver and low dosage has attracted lots of attention. ${ }^{13}$

PDGF has been found to play an essential role in wound repair and bone regeneration at early stage. ${ }^{\mathbf{1 4 , 1 5}}$ When released from activated platelets, PDGF acts as a chemotactic factor, recruiting bone mesenchymal stem cells (BMSCs) into the bone defect sites. ${ }^{15}$ Moreover, PDGF also induces the proliferation of BMSCs and endothelial cells. ${ }^{16}$ Therefore, we hypothesized that addition of PDGF may further accelerate simvastatin-induced bone regeneration process.

It must be noted that the expressions of various growth factors are temporal differences during natural bone regeneration. ${ }^{17}$ Thus, in order to obtain optimal osteogenesis, it is vital to simulate the temporal expression pattern of GFs when extrinsic osteogenic signals were applied. Core/shell microsphere is characterized with the distinct cored inner layer and a shelled outer layer. ${ }^{18}$ The core/shell features form two separate compartments, which the outer part (shell) encloses the inner part (core). Therefore, when incorporated in the core layer, the drugs will release with a controlled and sustained release profile due to the shell barrier. When the core and shell layers are composed of materials with low and high degraded rates respectively, the sequential release of drugs incorporated in core and shell layers will be achieved. ${ }^{15}$

In this study, we designed the core/shell microspheres to encapsulate simvastatin in core layer and PDGF in shell layer. Then, the release profiles of simvastatin and PDGF from core/ shell microspheres were investigated. Moreover, the osteogenic effects of simvastatin alone or with PDGF on critical-sized calvarial defect in rats were also explored.

\section{Materials and methods}

\subsection{Materials}

Poly-L-lactide (PLLA; MW = 85 000-160 000) and sodium alginate (300-400 cp) were purchased from Sigma-Aldrich (St. Louis, MO). Dichloromethane (DCM) and dimethyl sulfoxide (DMSO), bovine serum albumin (BSA) and simvastatin were obtained from SigmaAldrich (St. Louis, MO). Recombinant PGDF-BB, Spectro/Por membrane (molecular weight cut-off: 3500) were purchased from R\&D System (Minneapolis, MN) and Spectrum Laboratories (Rancho Domiguez, CA), respectively. For cultivation of cells, penicillin/streptomycin, high glucose Dulbecco's modified eagle's medium (DMEM) and fetal bovine serum (FBS) were purchased from Gibco (Life Invitrogen, CA). The 3-(4,5-dimethy2-thiazolyl)-2,5-diphenyl-2 $H$-tetrazolium bromide (MTT) was obtained from Sigma-Aldrich (St. Louis, MO).

\subsection{Fabrication of biomolecule-loaded core/shell microspheres}

The core/shell microspheres were formulated via CEHDA using PLLA and sodium alginate. In order to obtain the sustained release of simvastatin, the PLLA was used to compose core layer. Briefly, $500 \mathrm{mg}$ of PLLA was dissolved in $10 \mathrm{ml}$ DCM (core phase). Meanwhile, the aqueous alginate solution $(0.5 \%$; 300$400 \mathrm{cp}$ ) was obtained for the shell phase. To fabricate drugsloaded microspheres, the $1 \mathrm{mg}$ simvastatin was dissolved in the DCM with PLLA directly to form the inner core layer. For outer shell layer, 2 microgram $(\mu \mathrm{g})$ PDGF-BB was dissolved in aqueous alginate solution. For coaxial electrohydrodynamic atomization, two individual syringe pumps (KD Scientific, Holliston, MA) loaded PLLA/DCM (core phase) and aqueous alginate solution (shell phase) separately were transferred to the coaxial needle (Popper and Sons, Lake Success, NY) under an electrical field. Three types of microspheres were formulated: (1) blank microspheres without biomolecules, (2) simvastatin in core (M1), and (3) simvastatin in core and PDGF in shell (M2). The high voltage for the nozzle and ring supplied by voltage generator and was adjusted to produce the emerging droplets gradually until a stable Taylor cone jet was observed. To crosslink alginate, a grounded collector filled with $\mathrm{CaCl}_{2}(1 \%, \mathrm{w} / \mathrm{v})$ was placed at the distance of $10 \mathrm{~cm}$ under the nozzle. The microspheres were washed and isolated by the cell strainer. Then, the microspheres were sterilized by ethylene oxide, mixed with $\mathrm{CaCl}_{2}$ and stored at $-20{ }^{\circ} \mathrm{C}$ in tubes for future use. Microscopic light was used to observe the size and morphology of microspheres. The distribution of coumarin 6 in microspheres was detected by fluorescent microscope.

The SEM images of PLLA-alginate core/shell microspheres were obtained using ESEM FEI QUANTA 200F (FEI Corporation, Hillsboro, Oregon). The microspheres were cut in half, followed by coated within a thin layer of gold before observation. Samples were directly observed with dehydration. The observation was performed under $120 \mathrm{~Pa}$ at $-21^{\circ} \mathrm{C}$.

\subsection{Isolation of rat BMSCs and in vitro culture}

Rat BMSCs were harvested from the tibial and femoral marrows of 10 day old (Sprague-Dawley) SD rats. The animal surgery was carried out with the approval of the Second Xiangya Hospital of Central South University. Briefly, the rats were sacrificed by cervical dislocation and sterilized with ethyl alcohol. Then, the marrows of tibia and femur were flushed with DMEM. The mixture was collected and then centrifuged for $10 \mathrm{~min}$ at $1500 \mathrm{rpm}$. The supernate was discarded and BMSCs were then harvested. The BMSCs were suspended with DMEM supplemented with 10\% FBS and 1\% penicillin-streptomycin solution. The cells were cultured at $37{ }^{\circ} \mathrm{C}$ in $5 \% \quad \mathrm{CO}_{2}$ and a humidified atmosphere of 95\% air. After incubation for $24 \mathrm{~h}$, half of the medium was changed. The total medium was changed when cells were incubated for $48 \mathrm{~h}$. BMSCs from passages two to three were used for cell viability test.

\subsection{In vitro biocompatibility}

MTT assay was performed to estimate the cytotoxicity of prepared blank core/shell microspheres, M1 and M2 in vitro. Briefly, the indicated microspheres were immersed in DMEM for $24 \mathrm{~h}$ and the extracted media were prepared. The BMSCs were seeded at a density of $1 \times 10^{4}$ cells per well containing 
media with $10 \%$ FBS and incubated at $37^{\circ} \mathrm{C}$ for 1 day. Then, the cell culture media was removed, and the extracted media from indicated microspheres was added into each well with different extracted media concentrations $(0,25 \%, 50 \%$ and $100 \%)$ to culture another $72 \mathrm{~h}$. Then, $20 \mu \mathrm{l}$ MTT solution was added to each well. After incubation for $4 \mathrm{~h}$, the media was discarded and $200 \mu \mathrm{l}$ DMSO was added to each well. Under mild shaking, the 96-well plate was placed in enzyme-linked immunosorbent assay plate reader (Labsystems Multiskan, Labsystems, Finland) to measure the absorbance at $490 \mathrm{~nm}$.

\subsection{Encapsulation efficiency and release test in vitro}

To evaluate the encapsulation efficiency (EE) of simvastatin and PDGF in microspheres, $20.0 \mathrm{mg}$ of microspheres were dissolved in $1 \mathrm{ml} \mathrm{DCM}$, and then $1 \mathrm{ml}$ fresh PBS was introduced and centrifuged at $14000 \mathrm{rpm}$ for $3 \mathrm{~min}$. The aqueous layer was retrieved. The PDGF-BB concentration in aqueous solution was determined using ELISA kit, and the amounts of simvastatin in oil phase were measured by ultraviolet (UV) spectrophotometer at $242 \mathrm{~nm}$. The EE was calculated by

$\% \mathrm{EE}=$ mass of drug encapsulated $/$ mass of drug added $\times 100 \%$

For the in vitro release profile test, $10 \mathrm{mg}$ of indicated microspheres were placed in a Spectro/Por membrane bags, followed by stored into a tube with $10 \mathrm{ml}$ phosphate buffer saline (PBS, pH 7.4) at $37{ }^{\circ} \mathrm{C}$ under continuous stirring. At predetermined intervals, the whole volume of PBS in the tube was collected and replaced by fresh $10 \mathrm{ml}$ PBS. The concentration of released simvastatin and PDGF in PBS were quantified using UV spectrophotometer and ELISA kit, respectively. The cumulative percent release was obtained based on the normalization of released mass at specific time and the initial mass of biomolecules in microspheres at first.

\subsection{Animals}

Twenty nine male Sprague-Dawley rats (8 weeks old, 250-300 g) were used in this study. The care and treatment of rats was performed in compliance with relevant laws and guidelines established by the Institutional Animal Care and Use Committee of China. The animal research protocol was approved by the Laboratory Animal Care and Use Committee, Central South University.

\subsection{Surgical procedure}

The animals were anesthetized with an intraperitoneal injection of $10 \%$ chloral hydrate $\left(3 \mathrm{ml} \mathrm{kg}^{-1}\right)$. The surgical fields were shaved and scrubbed with iodine. A middle line incision in the sagittal plane across the cranium was performed, and a full thickness flap was elevated to expose the calvarial bone. A full-thickness, transosseous defect $(5 \mathrm{~mm}$ in diameter) was established on the cranium with a trephine drill under constant irrigation by saline. The rats with calvarial defects were randomly divided into the following groups $(n=6)$ : (1) a sham surgery control, (2) blank core/shell microspheres, (3) M1 (simvastatin-core/shell) and (4) M2 (simvastatin-core/PDGF-shell). The incisions were sutured with 4-0 silk in layers. A single intramuscular injection of cefazolin (30 $\mathrm{mg} \mathrm{kg}^{-1}$ ) was performed during the first 3 days postoperative days. Eight weeks after the surgery, the animals were sacrificed with an overdose of $10 \%$ chloral hydrate intraperitoneal injection. Bone specimens with the implanted microspheres were harvested and fixed with $4 \%$ paraformaldehyde for $24 \mathrm{~h}$.

\subsection{Microcomputed tomography}

Samples were analyzed using a micro-CT System (Sharp Corporation, Osaka, Japan). The relative bone volume (BV/TV), bone mineral density (BMD; $\mathrm{g} \mathrm{cm}^{-3}$ ) and the trabecular thickness (Tb.Th; $\mu \mathrm{m}$ ) of the volume of interests (VOI) in defects site were calculated using the micro-CT analysis software (Sharp Co., Osaka, Japan).

\subsection{Histological analysis}

The samples were decalcified with a 14\% EDTA for four weeks. Before sectioned into $5 \mu \mathrm{m}$ thick coronal serial sections through the center of defects site, the samples were dehydrated by the ethanol series with increasing concentration (70-100\%), cleared with xylene and embedded in paraffin. The $5 \mu \mathrm{m}$ thick sections were stained with hematoxylin and eosin (HE) as well as Masson staining. Subsequently, the stained sections were examined under light microscopy.

\subsection{Immunohistochemical assessment}

The expression of type I collagen was evaluated with the following protocol. Briefly, deparaffinised sections were first washed with PBS and blocked with $0.3 \%$ hydrogen peroxide for $20 \mathrm{~min}$, and then incubated with $5 \%$ bovine serum albumin (BSA). Subsequently, samples were incubated with primary antibody for type I collagen (Cell signaling Co., Beverly, USA) overnight at $4{ }^{\circ} \mathrm{C}$. After three times washed by PBS, samples were incubated with horseradish peroxidaseconjugated $\mathrm{ABC}$ for $20 \mathrm{~min}$, followed by incubation with biotinylated-conjugated secondary antibody (Sigma, St. Louis, USA) for another $20 \mathrm{~min}$. After that, addition of buffered 3,3diaminobenzidiinetetrahydrochloride (DBA) substrate was performed to visualize the immunostaining. Sample were stained with hematoxylin for $40 \mathrm{~s}$ each and dehydrated with the series ascending concentrations of ethanol solution, followed by cleared with xylene and covered with slips. For observation, all samples were detected under microscopic light (Olympus Co., Japan).

\subsection{Statistical analysis}

All data were presented as means \pm SD. Statistical analyses were carried out by one-way ANOVA. Tukey-Kramer Multiple Comparisons Test was performed in post hoc comparison. A $p$-value less than 0.05 was considered statistically significant. 


\section{Result}

\subsection{Identification the core/shell structures of microspheres}

Under an optimal condition, uniform microspheres composed of PLLA and alginate were successfully formulated. The core/ shell structures of microspheres were confirmed by inverted microscope (Fig. 1), characterized with transparent shell and translucent core. The interface between core and shell was distinct. Moreover, Fig. 2 showed that the core was stained into green by coumarin 6 . On the contrary, little staining of coumarin 6 was observed in the shell, which indicated the coumarin-6 did not diffuse into outer shell. Furthermore, after cutting apart, the microspheres were scanned with scanning electron microscopy. SEM images manifested the morphology and property of shell was different from that in core (Fig. 3).

\subsection{Encapsulation efficiency}

As displayed in Table 1, the EE of simvastatin in M1 and M2 were about $78 \%$ and $73 \%$, respectively. There were no significant differences in EE of simvastatin between M1 and M2. In the case of PDGF, the EE in M2 were about $65 \%$.

\subsection{Release profiles of simvastatin and PDGF in vitro}

Fig. 4 showed the release profiles of simvastatin and PDGF in vitro over 40 days. In the case of M1, simvastatin at core showed very minimal initial burst, only losing approximate $19 \%$ within the first 6 days, followed by release of about $85 \%$ within 40 days, which was characterized with a sustained and linear release model (Fig. 4a). As for drugs in M2, the release patterns of simvastatin and PDGF were markedly different due to the core/ shell wall, at least in partially. As anticipated, a strong initial burst of PDGF from shell was observed. At 6 day, more than $80 \%$ of PDGF was released, and then the PDGF continued its slow and little amount release, which reached a plateau by 9 days. On the contrary, simvastatin at the core showed a quite weak initial burst. The release pattern of simvastatin in M2 was similar with that in M1, indicating the little effect on simvastatin release after addition of PGDF in shell (Fig. 4b).

\subsection{Cytotoxicity of the biomolecules-loaded core/shell microspheres}

For estimating the biocompatibility alginate-PLLA microspheres, we performed MTT to test the cytotoxicity of

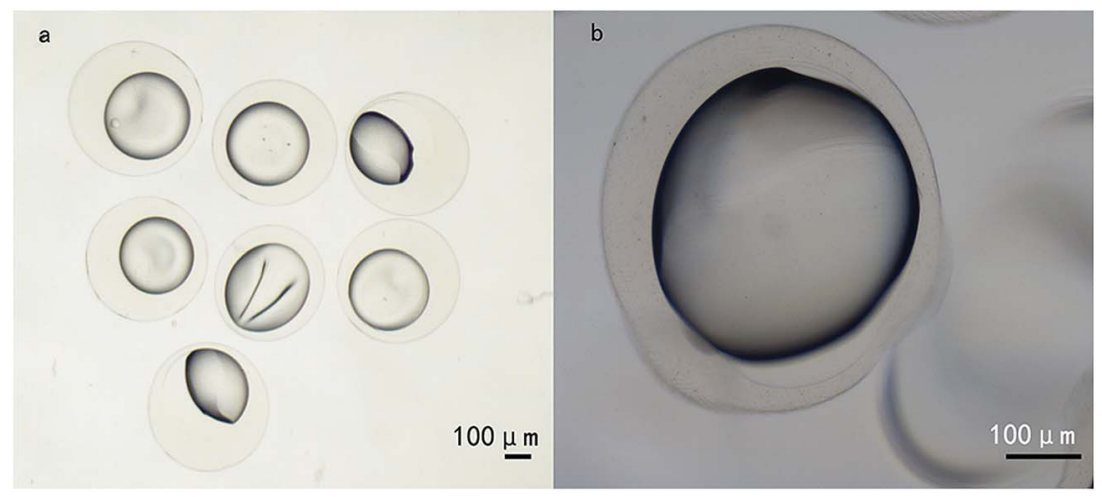

Fig. 1 Formation of core-shell microspheres using CEHDA in different magnification photomicrographs ((a) $\times 40$; (b) $\times 100)$. The CEHDA was effective in formulating microspheres with distinct core/shell structures. Measure bar $=100 \mu \mathrm{m}$.

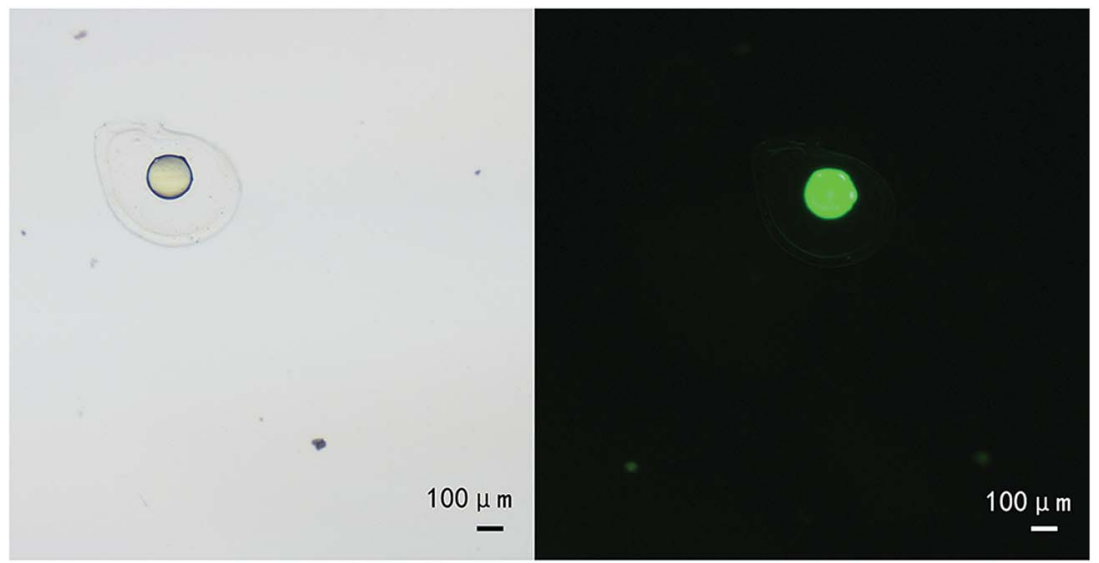

Fig. 2 The micrograph of core/shell microsphere (a) and fluorescence image of the same microsphere with coumarin 6-stained core compartment (b). Measure bar $=100 \mu \mathrm{m}$. 

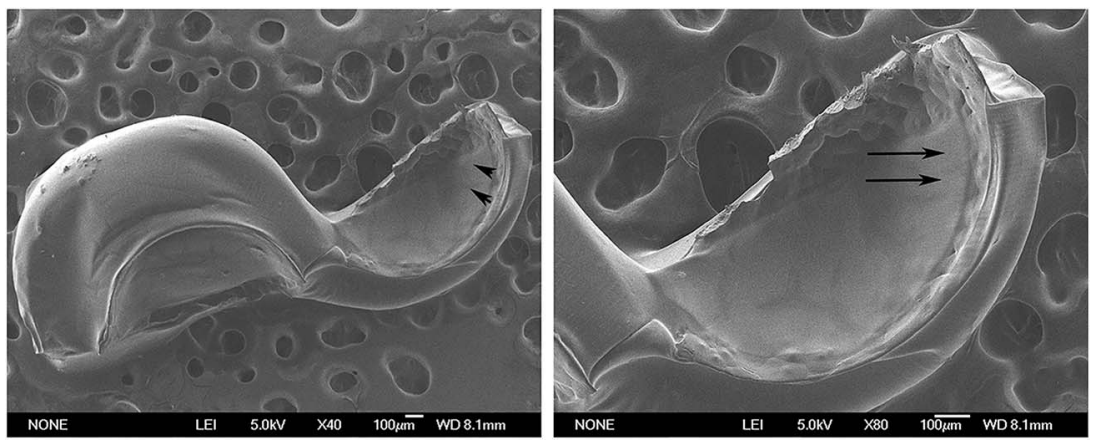

Fig. 3 The microspheres were cut apart and followed by scanning with SEM. The surface morphology of alginate shell was witness by SEM scan, a smooth surface was detected. On the contrary, the inner part marked with arrows showed a distinctly different texture in relative to alginate shell. Measure bar $=100 \mu \mathrm{m}$.

Table 1 The drug encapsulation efficiencies of PLLA/alginate microspheres with core/shell

\begin{tabular}{llll}
\hline & M1 & & M2 \\
\cline { 2 - 2 } & $\begin{array}{l}\text { Core: PLLA/simvastatin, } \\
\text { shell: alginate }\end{array}$ & $\begin{array}{l}\text { Core: PLLA/simvastatin, } \\
\text { shell: alginate/PDGF }\end{array}$ \\
\hline $\begin{array}{llll}\text { EE of } \\
\text { simvastatin (\%) } \\
\text { EE of PDGF (\%) }\end{array}$ & - & $73.24 \pm 3.80$ \\
& & $65.46 \pm 5.40$ & \\
\hline
\end{tabular}

microspheres in vitro. Indicated concentration of extracts from blank microspheres and M1 did not hamper BMSCs metabolism as well as proliferation (Fig. 5). Similarly, the OD values of cells incubated with $25 \%$ and $50 \%$ extracted media from M2 microspheres showed little difference when compared with that of control group. In contrast, OD value from cells cultured with $100 \%$ M2 extracted media were markedly greater than that of control group at $72 \mathrm{~h}(p<0.05)$.

\subsection{Results of the in vivo experiments}

The establishment of critical-sized rat calvarial defect was performed well. Within 5 or $7 \mathrm{~d}$ after surgery, 5 rats were subject to demise, 1 rat from control group, 2 rats from blank microspheres group and 1 rat from M1 group. The indicated rat calvarial defects were added for avoiding group bias. The skin over the bone defect site showed well healing without redness, swelling, infection or bone exposure throughout the wound healing period (data not show).

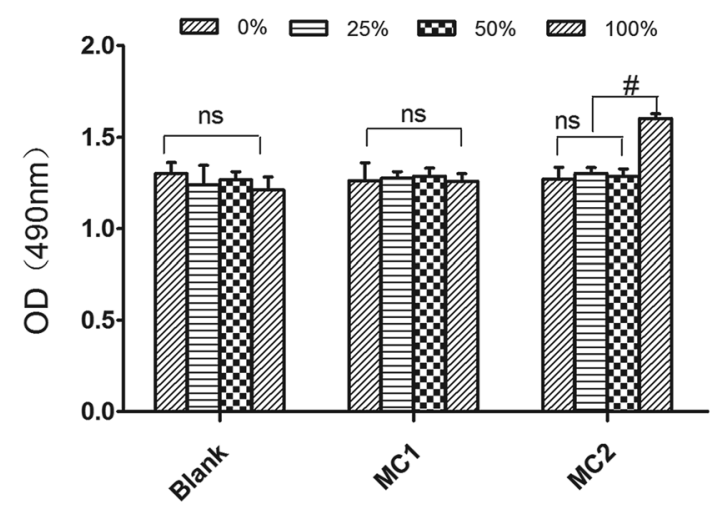

Fig. 5 Biocompatibility of indicated microspheres. Blank, microspheres without biomolecules; M1, microspheres with simvastatin in core layer; M2, microspheres with simvastatin in core layer and PDGF in shell layer. ns, no significant difference. $\# p<0.05 ; n=3$.

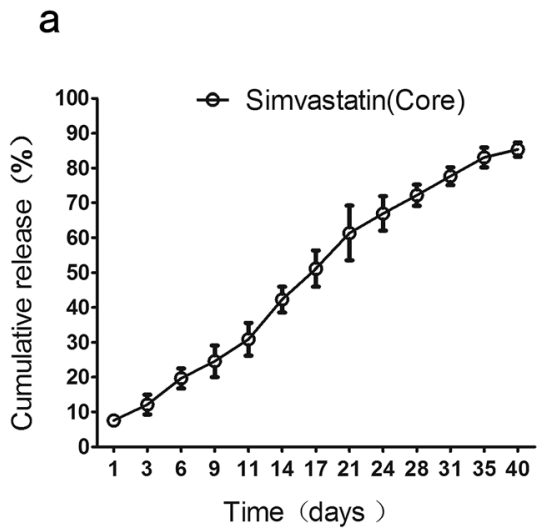

\section{b}

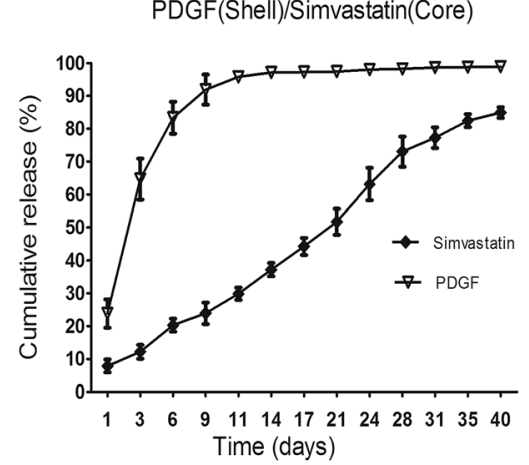

Fig. 4 The in vitro release profiles of simvastatin and PDGF from core/shell microspheres. (a) M1, simvastatin (core); (b) M2, simvastatin (core)PDGF (shell). PDGF, platelet-derived growth factor. 


\subsection{Micro-CT findings}

To investigate the effect of M1 and M2 on bone formation in vivo, the newly formed bone at each defect sites was assessed by three-dimensional reconstructed micro-CT at 8 weeks after surgery. Any radiopaque area located in the defect sites indicated the formation of new bone. As shown in Fig. 6a, both of thesham group and blank microsphere group showed little new bone formation. As expected, images from M1 group and M2 group exhibited considerable radiopaque areas, which suggested the new bone formation had extensively occurred.

For quantitative analysis, the bone mineral density (BMD), the ratio of mineralized $\mathrm{BV} / \mathrm{TV}$ and the trabecula thickness (Tb.Th) of newly formed bone were assessed by the computer analysis software (Fig. 6). The sham and blank microspheres groups revealed the lowest values in $\mathrm{BMD}, \mathrm{BV} / \mathrm{TV}$ and $\mathrm{Tb} . \mathrm{Th}$ among all experimental groups at 8 weeks. Indeed, these specific quantitative indicators of newly formed bone in blank microspheres group were similar to those in sham group at 8 weeks. However, compared with sham and blank microspheres groups, a markedly increase of BMD, BV/TV and Tb.Th were found in M1 and M2 groups. In addition, M2 group displayed a greater $\mathrm{BMD}$ value in defect site than M1 group $(p<0.01)$ (Fig. 6b). Similar trends were seen when analyzing the BV/TV and Tb.Th parameters in M1 and M2 groups (Fig. 6c and d).

\subsection{Histological observation and immunohistochemical evaluation histological observation}

At eight weeks, HE staining data from all samples showed little inflammation or infection. Both sham and blank microspheres groups showed limited bone formation. The defect sites of sham and blank microspheres groups were partly covered by fibrous tissue with a clear boundary of native bone. As expected, section from M2 group displayed extensive newly formed bone tissue and well-mineralized matrix deposition dispersed within the defect site. Some of the new bone fused into the margin of the defects, leaving a disappearing boundary between native bone and new bone tissue. At high magnification, some new regenerated bone was characterized with mature lamellar phenotype. As compared to sham and blank microspheres implanted group, moderate new bone matrix deposition was observe in defect area from M1 group. However, M1 group showed a significantly lesser bone formation than M2 group (Fig. 7).

Masson staining, which turns the collagen into blue-stained tissue, was utilized to assess the collagen regeneration. Results from Masson staining of the same sample supported the findings of HE staining. As illustrated in Fig. 8, collagen production

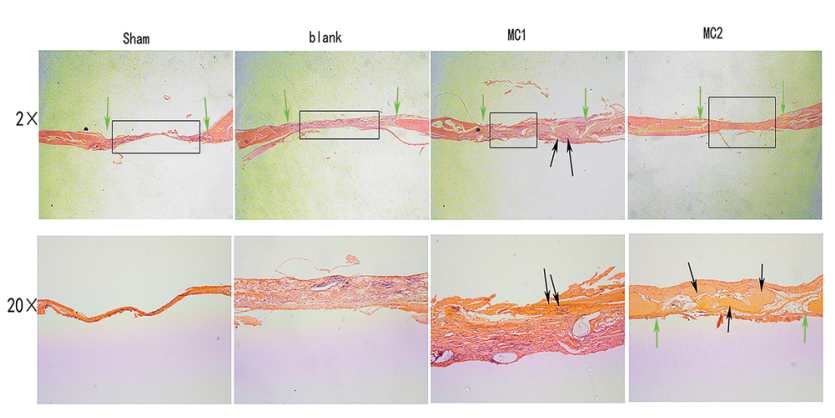

Fig. 7 Representative histological images of the defects at 8 weeks $(\mathrm{HE}$ stains, $\times 2, \times 20)$. Green arrow indicated to the original defect margins, black arrow indicated newly formed bone.
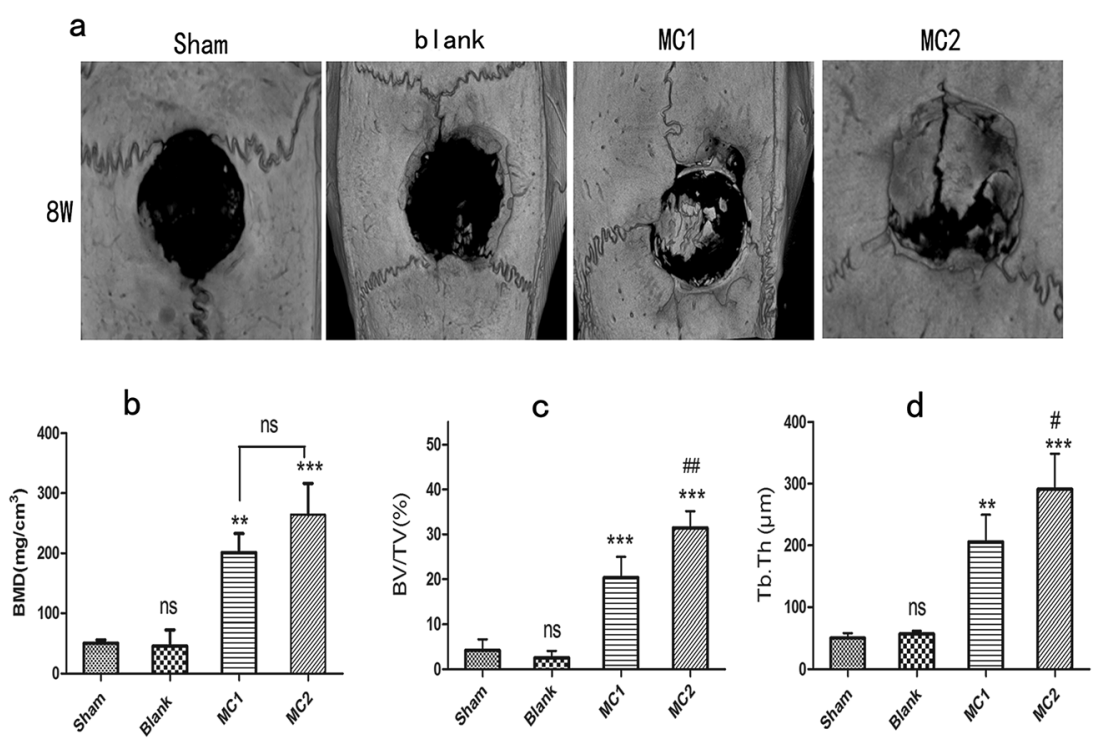

Fig. 6 Micro-CT analysis and quantitative evaluation of calvarial defects at 8 weeks after surgery. (a) Three-dimensional reconstructed micro-CT images of calvarial defects; (b) BMD; (c) Tb.Th; (d) BV/TV (data represent mean \pm standard deviation of six samples, and differences between groups were calculated by one-way ANOVA followed by Tukey-Kramer multiple comparisons test for post hoc comparisons. * statistically significant difference in relative to sham: $* p<0.05, * p<0.01, * * * p<0.001$; \# statistically significant difference in relative to M1: \#p<0.05, \#\#p< 0.01). Micro-CT, microcomputed tomography; BMD, bone mineral density; Tb.Th, trabecular thickness; BV/TV, the ratio of bone volume to total volume of the calvarial defects. 


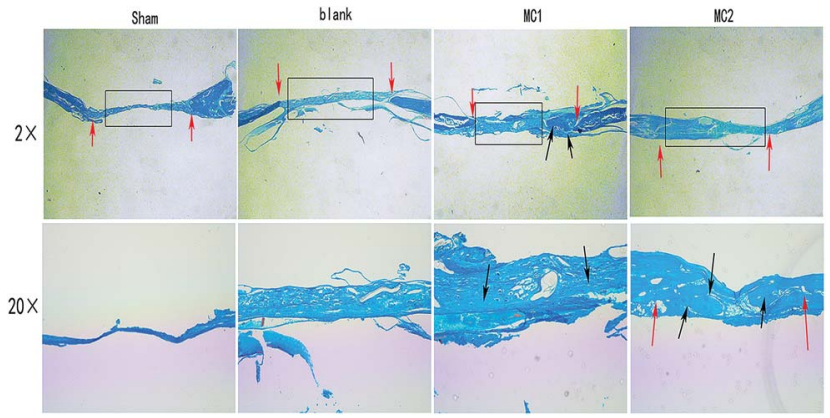

Fig. 8 Representative histological images of the Masson staining in defects $(\times 2, \times 20)$. Red arrow indicated to the original defect margins, and black arrow indicated newly produced collagen (stained in blue).

was rare in sham and blank microspheres group. However, abundant of collagen fibers were copiously secreted in M1 and M2 group. Moreover, collagen fibers in M2 group exhibited a more significant increase in production and greater alignment than M1 group.

\subsection{Immunohistochemical evaluation}

To further investigate the new bone formation promoted by M1 and M2 in rat calvarial defect, we performed immunohistochemical staining for type I collagen. Decalcified tissue

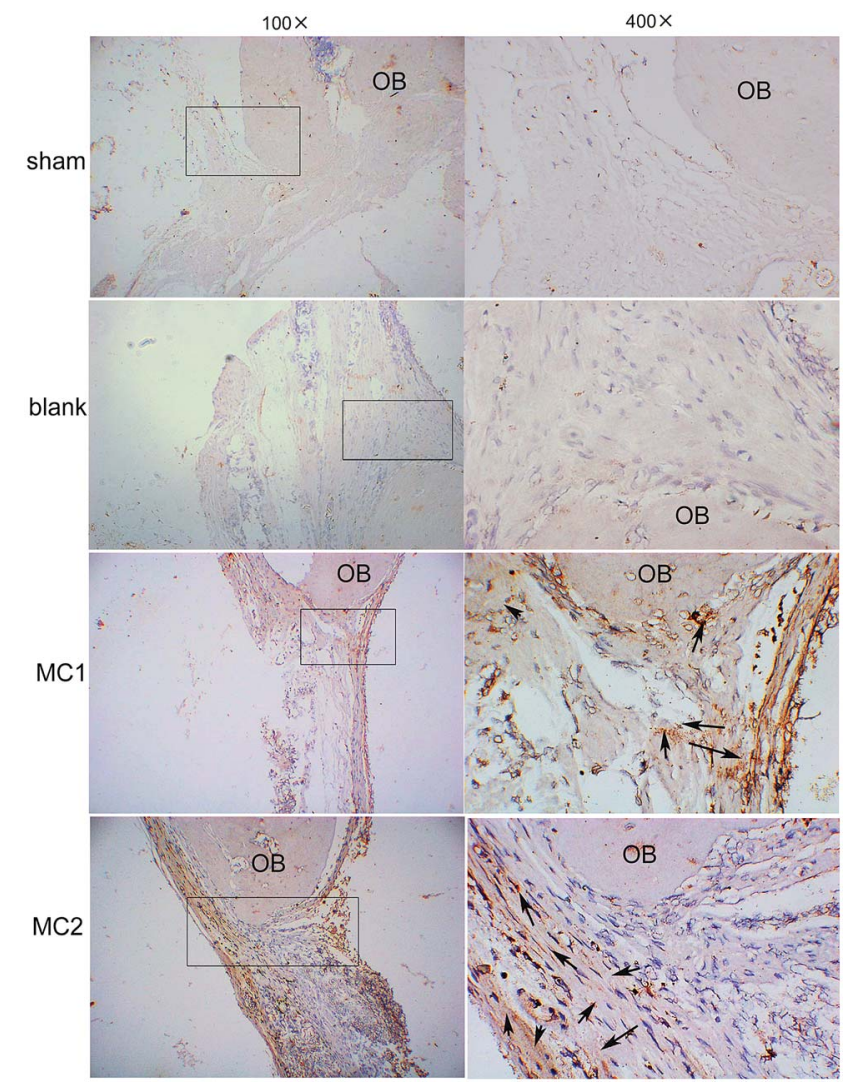

Fig. 9 Representative immunohistological measurement of type-I collagen in calvarial defects under different magnification photomicrographs $(\times 100, \times 400)$. Black arrow indicated the produced type-I collagen. OB, old bone. from sham and blank microspheres groups showed little staining for type I collagen. Compared to control and blank group, the staining for type I collagen had significantly greater intensity and area within the M1 and M2 groups. Notably, M2 groups exhibited a significantly higher staining for type I collagen in the matrix than M1 groups (Fig. 9).

\section{Discussion}

Bone regeneration is characterized by a highly coordinated biological process that is orchestrated with various events, including migration, proliferation and osteogenic differentiation of cells, with angiogenesis and remodeling of newly formed bone..$^{19}$ Specially, the osteogenic signals play the critical role in bone regeneration. ${ }^{4}$ Towards this end, we formulated the core/ shell microspheres as a drug delivery system to achieve the controlled release of simvastatin and PDGF, which greatly promoted bone regeneration at critical-sized calvarial defect in rat.

Since Mundy et al. first demonstrated that the simvastatin promoted bone formation in vivo in 1997, ${ }^{9}$ increasing reports have uncovered the pivotal roles of statins on bone regeneration. $^{20-22}$ Indeed, the effects of simvastatin on bone formation mainly relied on the way and dose of it administrated. The enhanced bone formation was only observed when oral administration of high-dose simvastatin at $20 \mathrm{mg}$ per $\mathrm{kg}$ per day in rats. Moreover, the bone mineral density at the lumbar and femoral neck was only increased when simvastatin at $40 \mathrm{mg}$ per day for one year was prescribed. ${ }^{23}$ However, some authors reported that simvastatin negative regulated bone formation in vivo. Maritz et al. uncovered that low-dose simvastatin at $1 \mathrm{mg}$ per $\mathrm{kg}$ per day inhibited new bone formation and facilitated bone resorption in rats. ${ }^{24}$ Furthermore, Nyan et al. demonstrated that inflammation in soft tissue and delayed bone union was elicited due to the burst release of local simvastatin. ${ }^{25}$ Accordingly, local controlled release of simvastatin in vivo is one of critical keys for successful application of simvastatin in new bone formation. For this purpose, we prepared the core/shell microspheres composed of PLLA and alginate through CEHDA. The CHEDA is a well-known fabrication method to produce disperse droplet, characterized with two coaxial nozzles. ${ }^{26}$ In this coaxial system, the two nozzles never merge, allowing the core and shell polymer flows in separate nozzles before dropping. With this design, the dual-walled microspheres with hydrophobic core and hydrophilic shell were formulated to delivery simvastatin and PDGF in one single step in our study.

In the present study, the simvastatin loaded in core showed a near zero-order release pattern in vitro. Before explaining the near zero-order release pattern of simvastatin in dual-walled microspheres, a better understanding of drugs release mechanism in one-walled microspheres was necessary. It is well accepted that the drugs in one-walled polymeric microspheres release mainly results from diffusion in early stage and polymer erosion in end stage. In one-walled microspheres, the initial burst release in early stage is mainly caused by the considerable diffusion of drugs at the microspheres surface. After that, the 
stable drugs release is due to the polymer erosion. In the last stage, the drugs release rates dropped because of depletion of drug. As a result, the drugs release profile is characterized with the typical sigmoid release pattern in one-walled microspheres. ${ }^{27}$ However, drugs release mechanism in dual-walled microspheres is entirely different. In core/shell microspheres, the simvastatin in PLLA core needs to diffuse through the shell to release into the environment. Due to the presence of shell layer being a physical barrier, the early diffusion rate of simvastatin is reduced. Moreover, as a protector, the alginate shell partly isolates the PLLA core from environment and slows down the rate of PLLA erosion, which decelerates the simvastatin release. ${ }^{28}$ Therefore, our study showed that the over-all simvastatin release rate was retarded by the shell layer and the nearzero release pattern was achieved. Consistently, Zhu et al. reported BMP2 encapsulated in a core/shell structure nanofibrous composed of polyethylene glycol and polycaprolactone expressed a zero order release for over 24 days in vitro. ${ }^{29}$

In contrast to the simvastatin release pattern, the PDGF in shell layer displayed a greatly strong initial burst release at early stage. Meanwhile, the depletion of PDGF in shell was observed within early 9 days in vitro. This phenomenon mainly attributed to the faster degradation of alginate in relative to PLLA. ${ }^{30}$ More importantly, the direct contact of shell layer and outer environment also contributed to the initial burst release of PDGF. Furthermore, the hydrophilic drugs (PDGF) usually release faster than hydrophobic drugs (simvastatin). Therefore, the simvastatin and PDGF expressed a sequential release pattern. Similar to this study, it has been reported that core/shell microspheres composed of PLLA and PLGA by CEHDA were able to achieve the sequential release of BMP-2 and FGF-2. The author demonstrated that the growth factor encapsulated in core compartment always exhibited much slower release rate in relative to that in shell compartment. ${ }^{31}$

The results from our animal experiments indicated that both simvastatin alone and simvastatin combined with PDGF in core/shell microspheres markedly stimulated new bone formation without inducing inflammation and scabbing in skin over defect area. On the contrary, little newly formed bone was observed in sham control and blank microspheres groups. Moreover, simvastatin combined with PDGF significantly increased bone formation compared to simvastatin alone. As simvastatin promotes bone formation mainly dependent on upregulation of BMP- $2,{ }^{32}$ the timing of simvastatin release could be critical to simulate the physiological expression pattern of BMP-2 during bone fracture healing. Bear in mind that the expression of BMP-2 is defined and induced immediately after fracture, followed by its peak expression at 2 weeks after bone injury, and then remains at a highly sustained level over 4 week period of rat fracture healing. ${ }^{17}$ In our study, the simvastatin in M1 and M2 exhibited a stable and continuously release profile within 40 days in vitro, which fitted well with the normal expression pattern of BMP-2 during bone healing. Meanwhile, due to the controlled release pattern, our study also showed that the $1 \mathrm{mg}$ simvastatin encapsulated in microspheres caused little inflammation in flap over defects area during healing, a finding that was consistent with previous study. ${ }^{33}$ In all, it is sustained released of simvastatin from core layer without obvious inflammatory response that, at least partly, contributed the considerable newly formed bone in our study.

It is widely accepted that bone regeneration is a complicated, coordinated process that regulated by several cytokines and growth factors. ${ }^{34}$ However, most reports were performed to observe the efficacy of delivery carriers encapsulating one single drug or growth factor on bone regeneration, which had some limitations. Therefore, a strategy that combined PDGF and simvastatin with core/shell microsphere was performed in our study. The three-dimensional micro-CT imagines of BMD, BV/ TV and Tb.Th were measured to quantify the strength and quality of neogenic bone. Our results demonstrated that all of BMD, BV/TV and Tb.Th in M2 were significantly improved in relative to M1. Moreover, immunohistochemical evaluation indicated that the type I collagen regeneration in M2 was strongly promoted than that in M1. These data indicate that simvastatin combined with PDGF delivery markedly increased new bone formation in relative to simvastatin alone. Moreover, Tb.Th was significantly increased in M2 groups than M1 groups, which suggested that PDGF addition promoted the calcium deposition and maturation of newly formed bone. It has been suggested that PDGF was able to recruit BMCs and endothelial cells near the defect sites, followed by increasing the proliferation of recruited cells. In addition, high level of PDGF activated macrophage to debride injury site and secret massive growth factors for bone regeneration. It has to be highlighted that the osteogenetic efficacy of PDGF mainly takes place in the early healing process. PDGF was released from platelets immediately after injury and mainly acted within the initial three day during bone regeneration. It is has been reported that persistent PDGF application suppressed the osteogenesis while short-term PDGF treatment enhanced the formation of mineralizing matrix. ${ }^{35} \mathrm{In}$ our study, PDGF release profile in M2 was characterized with substantially initial burst release, followed by near depletion within 6 days (more than 80\%), which coincided well with PDGF physiological release model in bone fracture healing. In this case, early released PDGF from shell layer recruited osteogenic cells, followed by increased proliferation (PDGF-induced mitogenesis), and then continuous released simvastatin from core layer enhanced osteogenic differentiation. Taken together, the sequential release pattern of PDGF and simvastatin contributed to improved new bone formation with better mechanical strength and quality in M2 than M1.

\section{Conclusion}

We successfully developed an ideal drug delivery system to achieve the controlled released of simvastatin and PDGF. Meanwhile, we demonstrated that simvastatin alone encapsulated in core/shell microspheres substantially promoted bone regeneration in vivo. Moreover, the synergistic osteogenesis was observed when simvastatin combined with PDGF. Therefore, these properties make PLLA-alginate core/shell microspheres the promising device for controlled and sequential drugs release and a beneficial strategy for patients who have bone defect in the near future. 


\section{Acknowledgements}

This study was founded by the grant of Xiangya famous doctors of Central South University. The authors confirm that there are no conflicts of interest associated with this publication.

\section{Notes and references}

1 E. Chiarello, M. Cadossi, G. Tedesco, P. Capra, C. Calamelli, A. Shehu and S. Giannini, Aging: Clin. Exp. Res., 2013, 25(1), S101-S103.

2 V. Devescovi, E. Leonardi, G. Ciapetti and E. Cenni, Chir. Organi Mov., 2008, 92, 161-168.

3 M. J. Pensak and J. R. Lieberman, Curr. Pharm. Des., 2013, 19, 3466-3473.

4 G. S. Anusuya, M. Kandasamy, R. S. Jacob, S. Sabarinathan, P. Ravishankar and B. Kandhasamy, J. Pharm. BioAllied Sci., 2016, 8, S39-S41.

5 W. F. McKay, S. M. Peckham and J. M. Badura, Int. Orthop., 2007, 31, 729-734.

6 B. Poon, T. Kha, S. Tran and C. R. Dass, J. Pharm. Pharmacol., 2016, 68, 139-147.

7 E. A. Naumenko, I. D. Guryanov, R. Yendluri, Y. M. Lvov and R. F. Fakhrullin, Nanoscale, 2016, 8, 7257-7271.

8 G. Biddeci, G. Cavallaro, F. Di Blasi, G. Lazzara, M. Massaro, S. Milioto, F. Parisi, S. Riela and G. Spinelli, Carbohydr. Polym., 2016, 152, 548-557.

9 G. Mundy, R. Garrett, S. Harris, J. Chan, D. Chen, G. Rossini, B. Boyce, M. Zhao and G. Gutierrez, Science, 1999, 286, 19461949.

10 C. Song, Z. Guo, Q. Ma, Z. Chen, Z. Liu, H. Jia and G. Dang, Biochem. Biophys. Res. Commun., 2003, 308, 458-462.

11 T. Maeda, A. Matsunuma, T. Kawane and N. Horiuchi, Biochem. Biophys. Res. Commun., 2001, 280, 874-877.

12 R. H. Jacobson, P. Wang and C. J. Glueck, JAMA, J. Am. Med. Assoc., 1997, 277, 296-297.

13 V. Bertolino, G. Cavallaro, G. Lazzara, M. Merli, S. Milioto, F. Parisi and L. Sciascia, Ind. Eng. Chem. Res., 2016, 55, 7373-7380.

14 P. Shah, L. Keppler and J. Rutkowski, Journal of Oral Implantology, 2014, 40, 330-340.

15 P. C. Chang, L. Y. Chong, A. S. Dovban, L. P. Lim, J. C. Lim, M. Y. Kuo and C. H. Wang, Tissue Eng., Part A, 2014, 20, 356364.

16 E. Canalis, S. Varghese, T. L. McCarthy and M. Centrella, Growth Regul., 1992, 2, 151-155.
17 T. J. Cho, L. C. Gerstenfeld and T. A. Einhorn, J. Bone Miner. Res., 2002, 17, 513-520.

18 R. A. Perez and H. W. Kim, Acta Biomater., 2015, 21, 2-19.

19 K. D. Hankenson, K. Gagne and M. Shaughnessy, Adv. Drug Delivery Rev., 2015, 94, 3-12.

20 J. Tan, N. Yang, X. Fu, Y. Cui, Q. Guo, T. Ma, X. Yin, H. Leng and C. Song, Med. Sci. Monit., 2015, 21, 1428-1439.

21 H. X. Zhang, G. Y. Xiao, X. Wang, Z. G. Dong, Z. Y. Ma, L. Li, Y. H. Li, X. Pan and L. Nie, J. Biomed. Mater. Res., Part A, 2015, 103, 3250-3258.

22 Y. Ayukawa, Y. Ogino, Y. Moriyama, I. Atsuta, Y. Jinno, M. Kihara, Y. Tsukiyama and K. Koyano, J. Oral Rehabil., 2010, 37, 123-130.

23 A. Montagnani, S. Gonnelli, C. Cepollaro, S. Pacini, M. S. Campagna, M. B. Franci, B. Lucani and C. Gennari, Bone, 2003, 32, 427-433.

24 F. J. Maritz, M. M. Conradie, P. A. Hulley, R. Gopal and S. Hough, Arterioscler., Thromb., Vasc. Biol., 2001, 21, 16361641.

25 M. Nyan, D. Sato, M. Oda, T. Machida, H. Kobayashi, T. Nakamura and S. Kasugai, J. Pharmacol. Sci., 2007, 104, 384-386.

26 H. Nie, Z. Dong, D. Y. Arifin, Y. Hu and C. H. Wang, J. Biomed. Mater. Res., Part A, 2010, 95, 709-716.

27 S. Freiberg and X. X. Zhu, Int. J. Pharm., 2004, 282, 1-18.

28 J. Wu, T. Kong, K. W. Yeung, H. C. Shum, K. M. Cheung, L. Wang and M. K. To, Acta Biomater., 2013, 9, 7410-7419.

29 H. Zhu, D. Yu, Y. Zhou, C. Wang, M. Gao, H. Jiang and H. Wang, J. Biomed. Mater. Res., Part B, 2013, 101, 541-552.

30 J. Venkatesan, I. Bhatnagar, P. Manivasagan, K. H. Kang and S. K. Kim, Int. J. Biol. Macromol., 2015, 72, 269-281.

31 L. Lei, S. Wang, H. Wu, W. Ju, J. Peng, A. S. Qahtan, C. Chen, Y. Lu, J. Peng, X. Zhang and H. Nie, J. Biomed. Mater. Res., Part A, 2015, 103, 252-261.

32 N. Horiuchi and T. Maeda, Oral. Dis., 2006, 12, 85-101.

33 I. C. Tai, Y. C. Fu, C. K. Wang, J. K. Chang and M. L. Ho, Int. J. Nanomed., 2013, 8, 3895-3904.

34 D. H. Kempen, L. B. Creemers, J. Alblas, L. Lu, A. J. Verbout, M. J. Yaszemski and W. J. Dhert, Tissue Eng., Part B, 2010, 16, 551-566.

35 S. C. Hsieh and D. T. Graves, J. Cell. Biochem., 1998, 69, 169180. 